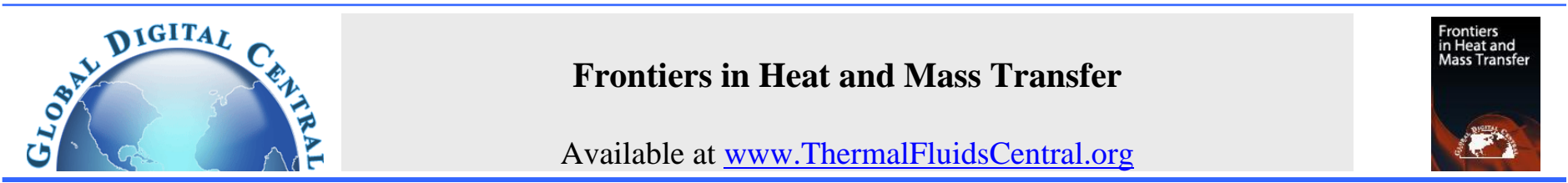

\title{
MIXED CONVECTION BOUNDARY LAYER FLOW OVER A VERTICALLY STRETCHING SHEET WITH CONVECTIVE BOUNDARY CONDITION AND EFFECT OF PARTIAL SLIP
}

\author{
Mitiku Daba*, P. Devaraj, S. V. Subhashini \\ Department of Mathematics, College of Engineering Guindy Campus, Anna University, Chennai, Tamil Nadu, Chennai-600 025, India
}

\begin{abstract}
In the present study, we investigated a problem of steady laminar mixed convection flow over a vertically stretching sheet with partial slip under convective surface boundary condition. The governing partial differential equations of the boundary layer flow are reduced into a set of nonlinear ordinary differential equations using a suitable similarity transformations. The system of non linear ordinary differential equations are solved by the Keller box method. Velocity, temperature and heat transfer rate are analyzed by considering the important parameters: Prandtl number $P r$, convective parameter $\epsilon$, slip parameter $K$ and mixed convection parameter $\lambda$ on the fluid flow and the corresponding results are presented graphically and discussed in detail. The investigation shows that surface heat transfer rate is affected by Prandtl number $P r$, slip parameter $K$ and mixed convection parameter $\lambda$. Increasing Prandtl number and mixed convection parameter $\lambda$ increases surface heat transfer rate but decreasing mixed convection parameter $\lambda$ and increasing the slip parametr $K$ reduces it. The numerical results obtained are compared with previously reported cases available from literature and they are found to be in very good agreement.

Keywords: Boundary layer. Mixed convection. Convective boundary condition. Keller box method. Partial slip.
\end{abstract}

\section{INTRODUCTION}

Mixed convection flow over a surface is a phenomenon having wider importance from both theoretical and practical situations due to its numerous applications in many engineering and geophysical areas. The processes occur when the effects of buoyancy forces in forced convection or the effects of forced flow in free convection become significant at the same time.

A free convection heat transfer due to the action of buoyancy and induced magnetic forces was investigated by Sparrow and Cess (1961) pointing out that presence of a magnetic field significantly affects the free convection heat transfer. Patil et al. (2009) considered a mixed convection flow over a continuously moving semi-infinite vertical plate due to the combined effects of thermal and mass diffusion by solving the coupled nonlinear partial differential equations numerically using implicit finite difference scheme in combination with quasilinearization. Boundary layer flow past a stretching plate have been studied by Ahmad et al. (2010) by using variable thermal conductivity to the flow problem and Aydin and Kaya (2009) analyzed the mixed convection flow of a viscous dissipating fluid about a vertical flat plate taking into account the effects of Ohmic heating and viscous dissipation. Bhattacharyya et al. (2013) examined effects of slip at the boundary on the mixed convective boundary layer flow over a flat plate. A problem of boundary layer flow over a stretching sheet in the presence of magnetic field has been solved by Pop and Tsung (1998). A similar work was done by Kumaran et al. (2010), who studied the transition effect of boundary layer flow due to an imposed and withdrawal of magnetic field over a viscous flow past a stretching sheet and have analyzed the problem numerically using one of the implicit finite difference methods called Crank-Nicholson. Later, Khan and Pop (2010) analyzed numerically the problem of laminar fluid flow past a stretching sheet in a nanofluid including the effects of Brownian motion and thermophoresis properties. A natural convection boundary-layer flow of nanofluid in a porous medium along a vertical plate be examined by Hady et al. (2011) wherein the problem has been solved numerically and analytically. Free convection and mass transfer flow over a vertical surface with the fluid considered to be electric conducting through a pours medium with constant heat flux in the presence of magnetic field is analyzed by Acharya, Dash, and Singh (2000) and Mansur and Ishak (2013) also studied the boundary layer flow of a nanofluid past a stretching/shrinking sheet with a convective boundary condition . Further, Bakar et al. (2012) extended the steady laminar flow over a stretching sheet with a convective boundary condition and considering the effect of partial slip. Most recently, Ali et al. (2014) discussed the problem of steady laminar magnetohydrodynamic (MHD) mixed convection stagnation-point flow of an incompressible viscous fluid over a vertical stretching sheet.

The flow over a stretching sheet has a wider applications in many industrial manufacturing processes such as the aerodynamic extrusion of plastic sheets and polymer extrusion, liquid film, hot rolling, wire drawing, paper production, drawing of plastic films, metal spinning, cooling

*Email: mitbru2007@yahoo.com 
of nuclear reactors during emergency shut down conditions, glass - fiber production and so on (Ferdows et al., 2012; Khan and Pop, 2010; Mahdy, 2012; Nadeem et al., 2013). This motivates us to study the problem of mixed convection flow over a stretching sheet. The aim of the present study is to investigate mixed convection boundary layer flow over a vertically stretching sheet with convective boundary condition and effect of partial slip. It is a new insight into such flow problems over a stretching sheet and also identifies the parameters that will affect the surface heat transfer rate. The boundary layer flow governed by the partial differential equations are first transformed into a system of nonlinear ordinary differential equations, before being solved numerically using Keller box method (Cebeci and Bradshaw, 1984; Na, 1979).

\section{MATHEMATICAL FORMULATION}

Consider a steady laminar mixed convection flow over a vertically stretching sheet of uniform surface temperature $T_{w}$ immersed in an incompressible viscous fluid and a Cartesian coordinate system is considered in which the $\mathrm{x}$-axis is measured along the plate in the upward direction and the $y$-axis is measured in the direction normal to the stretching sheet.

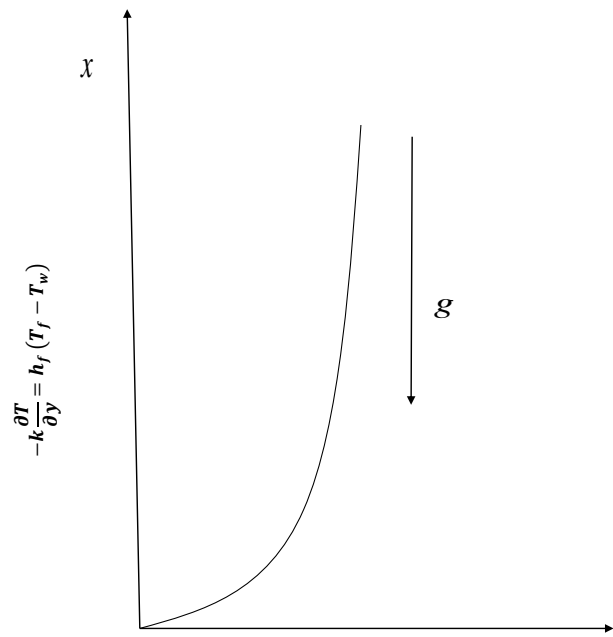

Fig. 1 Physical model and coordinate system

The sheet is stretched and its velocity is assumed to be $u_{w}=a x$, where $\mathrm{a}$ is a positive constant. The boundary-layer equations, under the Boussinesq approximation and for steady state flow conditions in the absence of heat generation and viscous dissipation, are given by:

$$
\begin{aligned}
\frac{\partial u}{\partial x}+\frac{\partial v}{\partial y} & =0 \\
u \frac{\partial u}{\partial x}+v \frac{\partial u}{\partial y} & =v \frac{\partial^{2} u}{\partial y^{2}}+g \beta\left(T-T_{\infty}\right) \\
u \frac{\partial T}{\partial x}+v \frac{\partial T}{\partial y} & =\alpha \frac{\partial^{2} T}{\partial y^{2}}
\end{aligned}
$$

with the boundary conditions:

$$
u=u_{w}+L \frac{\partial u}{\partial y} \text { and } v=0 \text { at } y=0 \text { and } u \rightarrow 0 \text { as } y \rightarrow \infty \text { for the }
$$
velocity field

$$
-k \frac{\partial T}{\partial y}=h_{f}\left(T_{f}-T_{w}\right) \text { at } y=0 \text { and } T \rightarrow T_{\infty} \quad \text { as } \quad y \rightarrow \infty \text { for }
$$
the thermal field, where $L$ is the proportionality constant of the velocity slip, $u$ and $v$ are the velocity components in the $\mathrm{x}$ and $\mathrm{y}$ directions respectively. We assume that the left surface of the sheet is heated by convection. We apply the following transformations to transform the nonlinear partial differential equations into a system of non linear ordinary differential equations.

$$
\eta=y\left(\frac{a}{\nu}\right)^{\frac{1}{2}}, \quad \psi(x, y)=(\nu a)^{\frac{1}{2}} f(\eta), \quad G(\eta)=\frac{T-T_{\infty}}{T_{f}-T_{\infty}} .
$$

The stream function $\psi(x, y)$ is defined as: $u=\frac{\partial \Psi}{\partial y}, v=-\frac{\partial \Psi}{\partial x}$. Continuity equation (1) is satisfied with the variable $\eta$ and the dimensionless stream function $f(\eta)$. Using the similarity transformations and the function $f(\eta)$, equation (2) and (3) respectively reduce to the following ordinary differential equations:

$$
\begin{aligned}
f^{\prime \prime \prime}+\frac{f}{2} f^{\prime \prime}-f^{\prime 2}+\lambda G & =0 \\
G^{\prime \prime}+\operatorname{Pr} f G^{\prime} & =0
\end{aligned}
$$

In order to get similarity solution we take $h_{f}=\frac{c}{\sqrt{x}}$, where $\mathrm{c}$ is constant. The transformed boundary conditions can be written as :

$$
\begin{array}{r}
f^{\prime}(0)=1+K f^{\prime \prime}(0), \quad f(0)=0, G^{\prime}(0)=-\epsilon(1-G(0)), \\
f^{\prime} \rightarrow 0, \quad G \rightarrow 0 \text { as } \eta \rightarrow \infty,
\end{array}
$$

where $K=L \sqrt{\frac{a}{\nu}}, \epsilon=\frac{c}{k} \sqrt{\frac{\nu}{u_{\infty}}}$ and $\lambda=\frac{g \beta\left(T_{f}-T_{\infty}\right)}{a^{2} L}$.

The physical quantities of interest are the local skin friction $C_{f}$ and the local Nusselt number $N u_{x}$ which are defined as:

$$
\begin{aligned}
C_{f} & =\frac{2 \tau_{w}}{\left(\rho u_{w}^{2}\right)} \\
N u_{x} & =\frac{x q_{w}}{k\left(T_{f}-T_{\infty}\right)},
\end{aligned}
$$

where the surface shear stress $\tau_{w}$ and the surface heat flux $q_{w}$ are given by

$$
\tau_{w}=\left.\mu \frac{\partial u}{\partial y}\right|_{y=0}, \text { and } q_{w}=-\left.k \frac{\partial T}{\partial y}\right|_{y=0} .
$$

Using the similarity variable, equation (7) and (8) respectively becomes

$$
\frac{1}{2} C_{f} \sqrt{R e_{x}}=f^{\prime \prime}(0), \quad \frac{N u_{x}}{\sqrt{R e_{x}}}=-G^{\prime}(0),
$$

where $R e_{x}=\frac{U_{w} x}{\nu}$ is the Reynolds number.

\section{RESULTS AND DISCUSSION}

The non linear ordinary differential equations (4) and (5) with the boundary condition (6) were solved numerically by Keller box method. The computations have been carried out for different values of Prandtl number $\operatorname{Pr}$, convective parameter $\epsilon$, slip parameter $K$ and mixed convection parameter $\lambda$. Figures $2-9$ show the effect of these parameters on velocity \& temperature profiles and Figures $10-12$ discuss the effect of the parameters on surface heat transfer rate. In order to validate our method, we have compared steady state results of heat transfer coefficient $G^{\prime}(0)$, $\eta_{\delta}$ and $\eta_{\infty}$ with those of the works by Cortell (2008) and Anilkumar (2011). Where, $\eta_{\delta}$ is non-dimensional thickness of the boundary layer and $\eta_{\infty}$ is the value of $\eta$ at the free stream when the flow is the boundary layer flow on a flat plate with no effect of convective parameter $\epsilon$, slip parameter $K$ and mixed convection parameter $\lambda$. The comparison is given in Table 1.

Figures 2 and 3 depict the effect of mixed convection parameter $\lambda$ and Prandtl number $\operatorname{Pr}$ on velocity profile keeping other parameters constant. We see from these figures that there is no significant effects of $\lambda \& \operatorname{Pr}$ on velocity of the fluid. The velocity profile decreases as the slip parameter $K$ increases as it is interpreted by Figure 4. From this we can deduce that as $K$ increases the skin coefficient friction $f^{\prime \prime}(0)$ decreases in magnitude. Figure 5 explains the relationship between convective parameter $\epsilon$ and the non-dimensional temperature. As indicated in the figure, when the value of convective parameter $\epsilon$ getting large the temperature also rises. This is to mean that temperature of the hot fluid which is found to the left of the sheet is convicted to the right fluid. This causes the surface temperature to rise and consequently increases the temperature of the fluid which is found to the right of the stretching sheet. The reverse 
Table 1 Comparison of $G^{\prime}(0), \eta_{\delta}$ and $\eta_{\infty}$ with those of the works by Cortell (2008) and Anilkumar (2011) with no effect of $\epsilon, K$ and $\lambda$.

\begin{tabular}{|l|l|l|l|l|l|l|}
\hline & \multicolumn{3}{|c|}{ Cortell (2008) } & \multicolumn{3}{c|}{ Anilkumar (2011) } \\
\hline $\operatorname{Pr}$ & $G^{\prime}(0)$ & $\eta_{\delta}$ & $\eta_{\infty}$ & $G^{\prime}(0)$ & $\eta_{\delta}$ & $\eta_{\infty}$ \\
\hline 0.7 & 0.29268 & 5.64 & 8.36 & 0.29268 & 5.64 & 8.36 \\
5 & 0.57669 & 2.75 & 4.07 & 0.57668 & 2.74 & 4.07 \\
10 & 0.72814 & 2.17 & 3.22 & 0.72812 & 2.16 & 3.20 \\
30 & 1.05173 & 1.50 & 2.24 & 1.05171 & 1.50 & 2.22 \\
50 & 1.24729 & 1.27 & 1.90 & 1.24729 & 1.26 & 1.91 \\
100 & 1.57183 & 1.01 & 1.52 & 1.57181 & 1.00 & 1.52 \\
1000 & 3.38709 & 0.46 & 0.72 & - & - & - \\
\hline \hline \multicolumn{7}{|c|}{ Present } \\
\cline { 1 - 4 } $\operatorname{Pr}$ & $G^{\prime}(0)$ & $\eta_{\delta}$ & $\eta_{\infty}$ & & & \\
\hline 0.7 & 0.29268 & 5.64 & 8.36 & & & \\
5 & 0.57670 & 2.75 & 4.07 & & & \\
10 & 0.72816 & 2.17 & 3.22 & & \\
30 & 1.05173 & 1.50 & 2.24 & & \\
50 & 1.24736 & 1.27 & 1.90 & & \\
100 & 1.57197 & 1.00 & 1.51 & & \\
1000 & 3.38843 & 0.47 & 0.72 &
\end{tabular}

situation also happen when the right hand side is assumed to be hot as it is shown by Figure 6 .

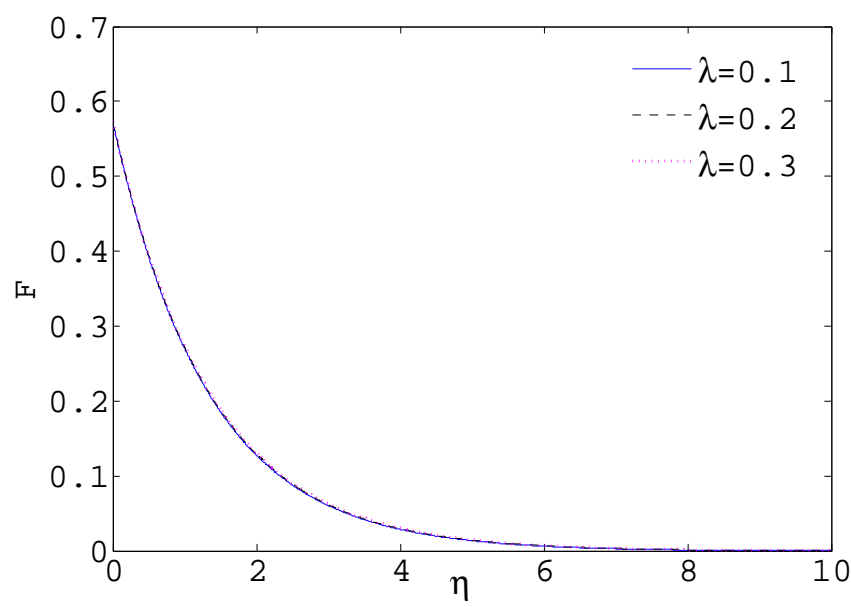

Fig. 2 Velocity profile for some values of $\lambda$

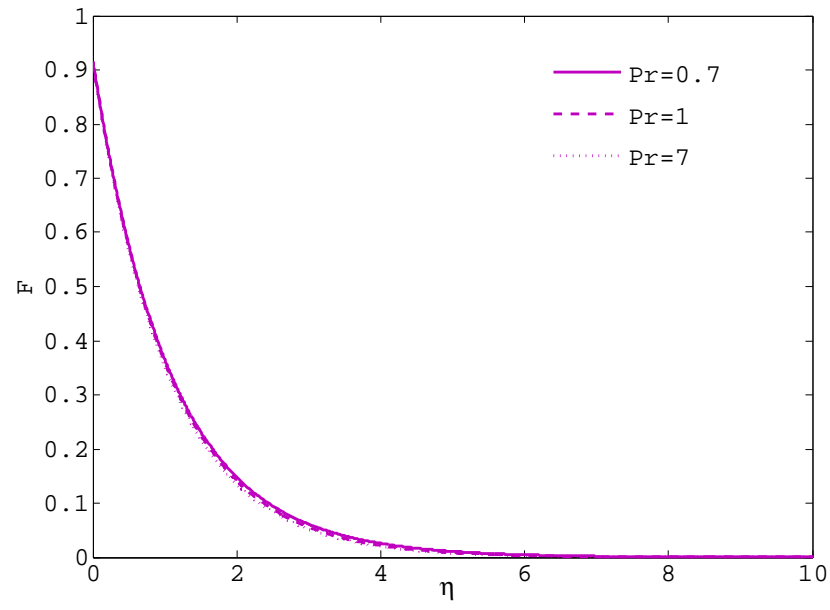

Fig. 3 Velocity profile for some values of Prandtl number

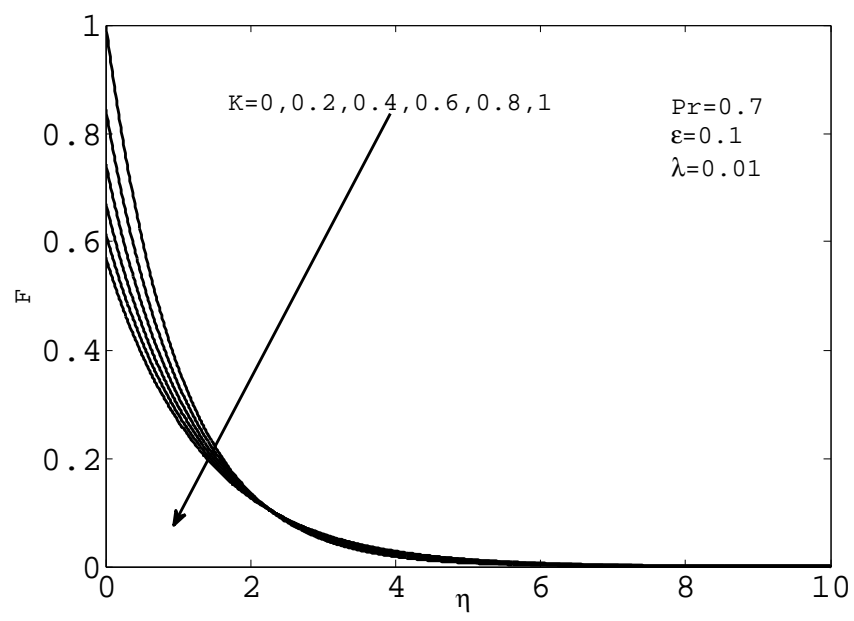

Fig. 4 Velocity profile for different values of slip parameter $K$

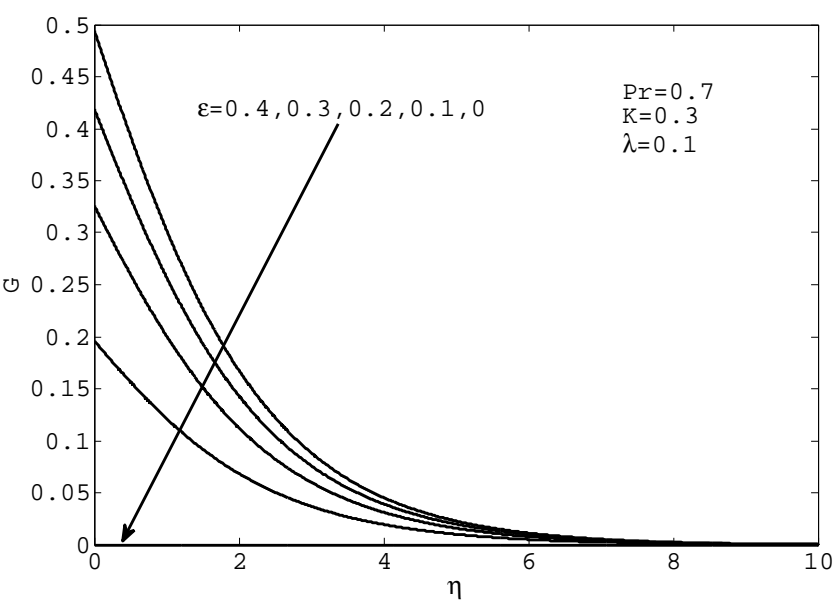

Fig. 5 Effect of convective parameter $\epsilon$ on temperature profile

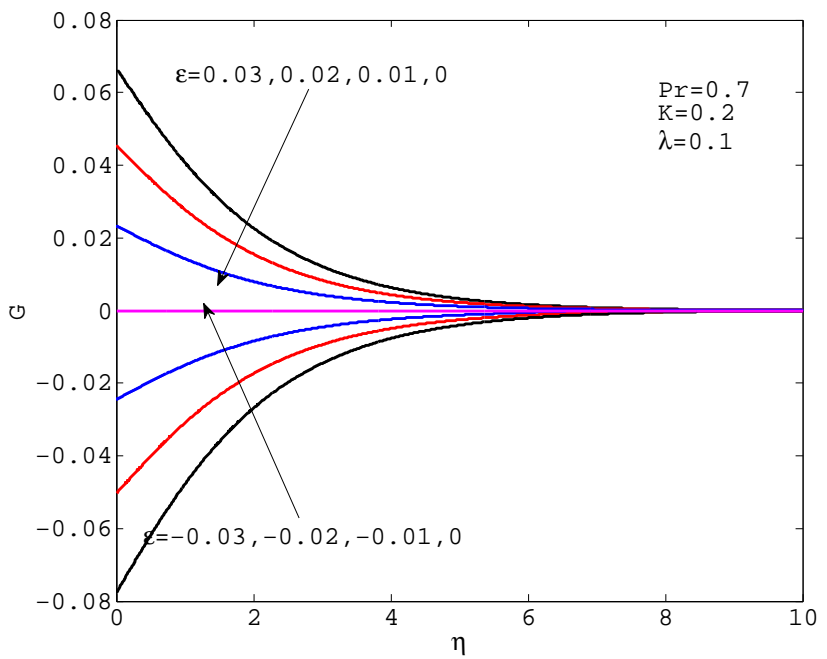

Fig. 6 Effect of convective parameter $\epsilon$ on temperature profile 


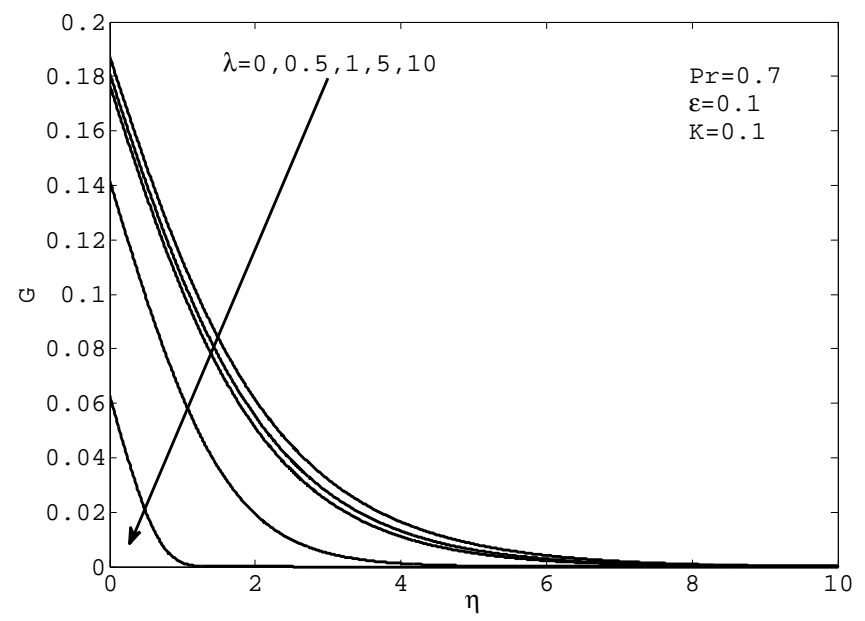

Fig. 7 Effect of mixed convection parameter $\lambda$ on temperature profile

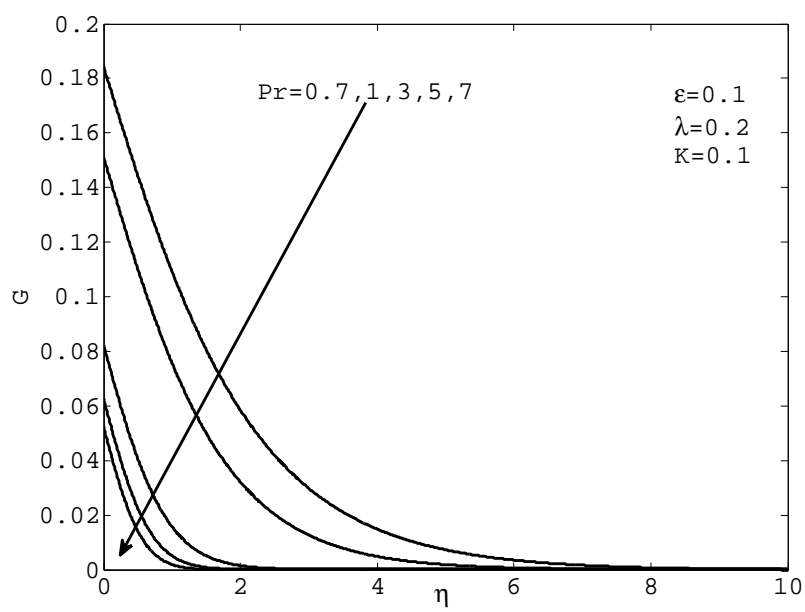

Fig. 8 Effect of Prandtl number on temperature profile

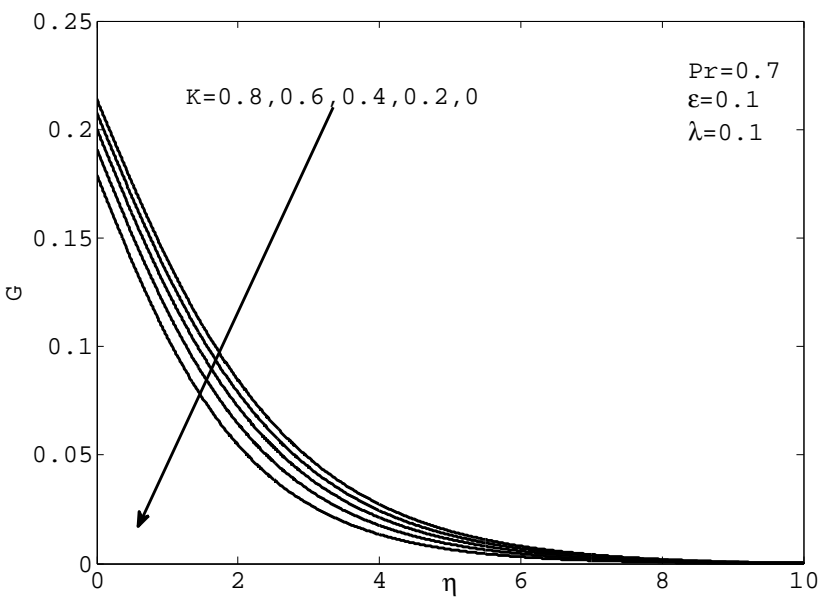

Fig. 9 Temperature profile for different values of $K$

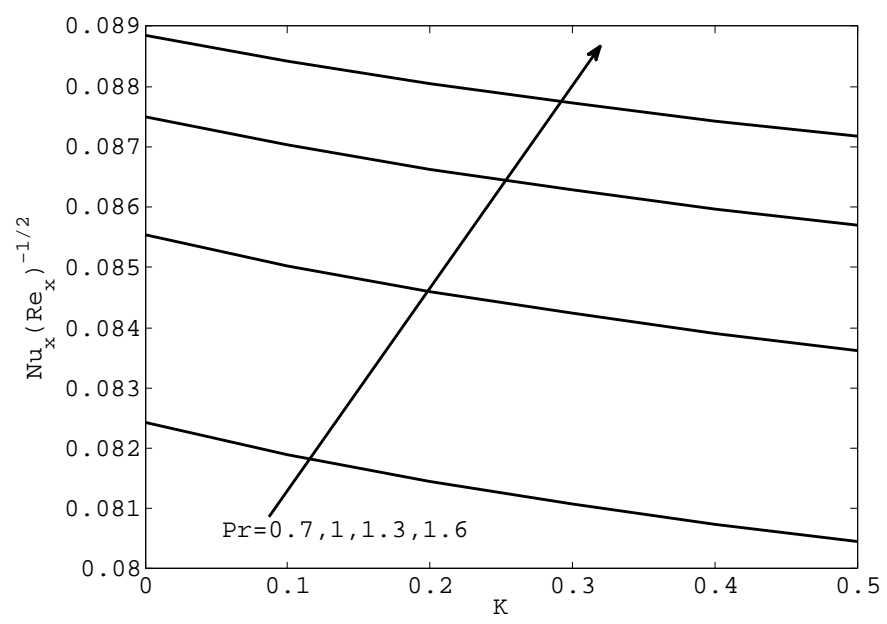

Fig. 10 Effect of Prandtl number $P r$ and slip parameter $K$ on surface heat transfer rate when $\epsilon=0.1$ and $\lambda=0.5$

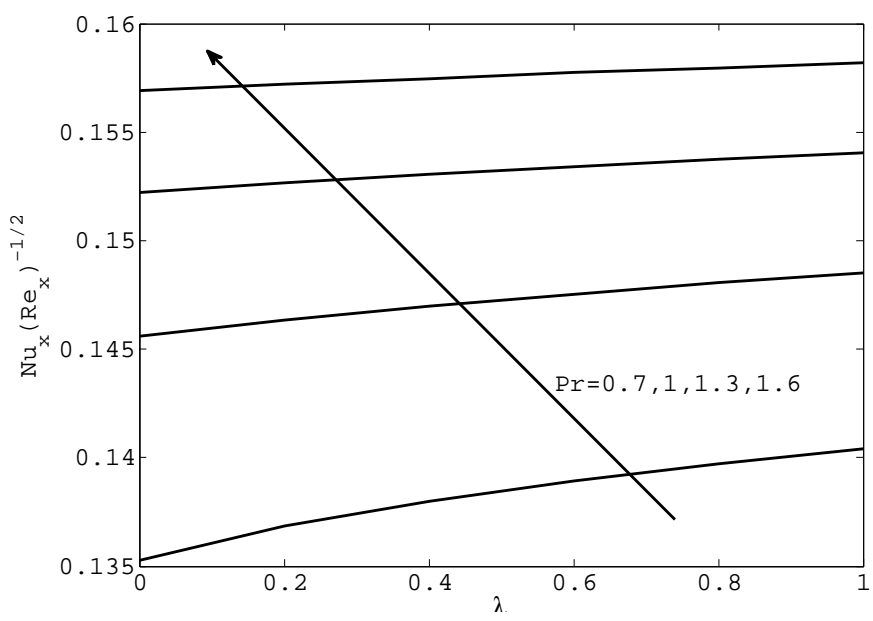

Fig. 11 Effect of Prandtl number $\operatorname{Pr}$ and mixed convection parameter $\lambda$ on surface heat transfer rate when $\epsilon=K=0.2$

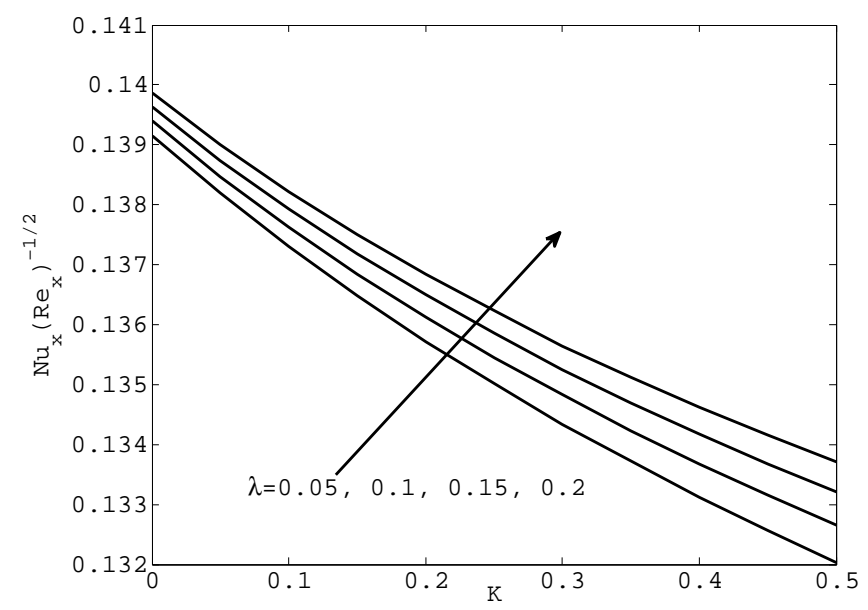

Fig. 12 Effect of mixed convection parameter $\lambda$ and slip parameter $K$ on surface heat transfer rate when $\epsilon=0.2, P r=0.7$ 
Figure 7 illustrates that when the mixed convection parameter $\lambda$ increases the thermal boundary layer getting thick. Consequently this reveals that the surface temperature gradient increases in magnitude in which this produces an increase in the surface heat transfer rate. Physically $\lambda>0$ means that it is assisting flow and which means heating of the fluid or cooling of the surface of the stretching sheet. Effect of Prandtl number on temperature profile is depicted in Figure 8. According to the figure, as the Prandtl number Pr increases the thermal boundary layer is getting thin. This is because a higher Prandtl number fluid has relatively low thermal conductivity which reduces conduction. It is also evident from the figure that temperature gradient at the surface increases in magnitude as the Prandtl number increases. This indicates that there is an increase in the surface heat transfer rate. A correlation between the dimensionless temperature $\mathrm{G}$ and slip parameter $K$ is presented on Figure 9. The figure tells us that as the slip parameter $\mathrm{K}$ increases the surface heat transfer rate decreases. Which implies that the dimensionless temperature tends to rise up. Influence of Prandtl number and slip paramter $K$ on a local Nusselt number named as surface heat transfer rate is displayed in Figure 10. It is seen from the figure that decreasing the Prandtl number and increasing the slip parameter $K$ will result in a decrease of the surface heat transfer rate. But increasing the Prandtl number and the mixed convection parameter $\lambda$ increases the surface heat transfer rate as it is shown by Figure 11 . Impact of mixed convection parametr $\lambda$ and the slip parameter $K$ on surface heat transfer rate is described by Figure 12 . It is revealed by the figure that decreasing the mixed convection parameter $\lambda$ and increasing the slip parameter $K$ is to decrease the surface heat transfer rate.

\section{CONCLUSIONS}

We studied the problem of steady mixed convection boundary layer flow over a vertically stretching sheet in a viscous incompressible fluid with convective surface boundary condition and partial slip. The governing partial differential equations are transformed into ordinary differential equations using similarity transformation, which are more convenient for numerical computation. The transformed ordinary differential equations are then solved numerically by the Keller box method. The numerical results obtained are compared with previously reported cases available from literature and they are found to be in very good agreement. The effect of Prandtl number $\mathrm{Pr}$, convective parameter $\epsilon$, slip parameter $\mathrm{K}$ and mixed convection parameter $\lambda$ on velocity, temperature and surface heat transfer rate are presented. From the present investigation, we found that:

- Effects of Prandtl number $\operatorname{Pr}$ and mixed convection parameter $\lambda$ on velocity profiles are insignificant.

- Raising the Prandtl number or mixed convection parameter $\lambda$ tends to increase the surface heat transfer rate.

- The velocity profile decreases as the slip parameter $K$ increases.

- Rise of the temperature can be observed in an increase of convective parameter $\epsilon$.

- A decrease of Prandtl number $P r$ and an increase of slip parameter $K$ will result in a decrease of surface heat transfer rate.

- Increasing Prandtl number and mixed convection parameter $\lambda$ increases the surface heat transfer rate but decreasing the mixed convection parameter $\lambda$ and increasing the slip parametr $K$ reduces it.

\section{ACKNOWLEDGEMENTS}

The first author would like to thank Indian Council for Cultural Relations, Ministry of External Affairs, Govt. of India for providing the research fellowship to carry out the research work.

\section{NOMENCLATURE}

$C_{f} \quad$ local skin friction coefficient

$c_{p} \quad$ specific heat capacity

$F \quad$ dimensionless velocity

$f \quad$ dimensionless stream function

$G \quad$ dimensionless temperature

$h_{f} \quad$ heat transfer coefficient

$k \quad$ thermal conductivity

$K \quad$ slip parameter

$L \quad$ proportionality constant of the velocity slip

$N u_{x} \quad$ local Nusselt number

$\operatorname{Pr} \quad$ Prandtl number

$q_{w} \quad$ surface heat flux

$R e_{x} \quad$ local Reynolds number

$T$ temperature of the fluid

$T_{f} \quad$ temperature of the hot fluid

$T_{\infty} \quad$ temperature of the fluid far away from the wall

$T_{w} \quad$ temperature at the wall

$u \quad$ velocity component in $\mathrm{x}$-direction

$U_{w} \quad$ stretching sheet wall velocity

$v \quad$ velocity component in y-direction

Greek Symbols

$\alpha \quad$ thermal diffusivity

$\nu \quad$ kinematic viscosity

$\mu \quad$ dynamic viscosity

$\rho \quad$ density of the fluid

$\eta \quad$ transformed variable

$\psi \quad$ stream function

$\tau_{w} \quad$ wall shear stress

$\lambda \quad$ mixed convection parameter

$\epsilon \quad$ convective parameter

\section{REFERENCES}

Acharya, M., Dash, G., and Singh, L., 2000, "Magnetic Field Effects On The Free Convection And Mass Transfer Flow Through Porous Medium With Constant Suction And Constant Heat Flux," Indian Journal of Pure and Applied Mathematics, 31(4), 1-18.

Ahmad, N., Siddiqui, Z., and Mishra, M., 2010, "Boundary Layer Flow And Heat Transfer Past A Stretching Plate With Variable Thermal Conductivity," International Journal of Non-Linear Mechanics, 45, 306-309. http://dx.doi.org/10.1016/j.ijnonlinmec.2009.12.006.

Ali, F., Nazar, R., Arifin, N., and POP, I., 2014, "Mixed Convection Stagnation-Point Flow on Vertical Stretching Sheet with External Magnetic field," Applied Mathematics and Mechanics (English Edition), 35(2), 155-166.

http://dx.doi.org/10.1007/s10483-014-1780-8.

Anilkumar, D., 2011, "Nonsimilar Solutions for Unsteady Mixed Convection From a Moving Vertical Plate," Communications in Nonlinear Science and Numerical Simulation, 16, 3147-3157.

http://dx.doi.org/10.1016/j.cnsns.2010.11.017.

Aydin, O., and Kaya, A., 2009, "MHD Mixed Convection of a Viscous Dissipating Fluid about a Permeable Vertical Flat Plate," Mathematical Modelling, 33, 4086-4096.

http://dx.doi.org/10.1016/j.apm.2009.02.002.

Bakar, N., Zaimi, W., Hamid, R., Bidin, B., and Ishak, A., 2012, "Boundary Layer Flow over q Stretching Sheet with a Convective Boundary Condition and Slip Effect," World Applied Sciences Journal, 17, 49-53. 
Bhattacharyya, K., Mukhopadhyay, S., and Layek, G., 2013, "Similarity Solution Of Mixed Convective Boundary Layer Slip Flow Over A Vertical Plate," Ain Shams Engineering Journal, 4, 299-305.

http://dx.doi.org/10.1016/j.asej.2012.09.003.

Cebeci, T., and Bradshaw, P., 1984, Physical and Computational Aspects of Convective Heat Transfer, Springer-Verlag, New York.

Cortell, R., 2008, "Radiation effects in the Blasius flow," Applied Mathematics And Computation, 198, 333-338.

http://dx.doi.org/10.1016/j.amc.2007.08.037.

Ferdows, M., Khan, M., Mahmud, M., and Shuyu, S., 2012, "MHD mixed convective boundary layer flow of a nanofluid through a porous medium due to an exponentially stretching sheet," Mathematical Problems in Engineering.

http://dx.doi.org/10.1155/2012/408528.

Hady, F., Ibrahim, F., M.R., S.A.G., and Eid, 2011, "Boundary-layer Non-Newtonian Flow Over Vertical Plate In Porous medium saturated with nanofluid," Applied Mathematics and Mechanics (English Edition), 32(12), 1577-1586.

http://dx.doi.org/10.1007/s10483-011-1524-7.

Khan, W., and Pop, I., 2010, "Boundary-layer Flow of a Nanofluid Past a Stretching Sheet," International Journal of Heat and Mass Transfer, 53, 2477-2483.

http://dx.doi.org/10.1016/j.ijheatmasstransfer.2010.01.032.

Kumaran, V., Kumar, A., and Pop, I., 2010, "Transition Of MHD Boundary Layer Flow Past P Stretching Sheet," Commun Nonlinear Sci Numer Simulat, 15, 300-311.

http://dx.doi.org/10.1016/j.cnsns.2009.03.027.
Mahdy, A., 2012, "Unsteady mixed convection boundary layer flow and heat transfer of nanofluids due to stretching sheet," Nuclear Engineering and Design, 249, 248âĂŞ255.

http://dx.doi.org/10.1016/j.nucengdes.2012.03.025.

Mansur, S., and Ishak, A., 2013, "The Flow and Heat Transfer af a Nanofluid Past a Stretching or Shrinking Sheet with a Convective Boundary Condition," Abstract and Applied Analysis. http://dx.doi.org/10.1155/2013/350647.

$\mathrm{Na}, \mathrm{T} ., 1979$, Computational methods in engineering boundary balue problems, Academic Press, New York.

Nadeem, S., Haq, R., Akbar, N., Lee, C., and Khan, Z., 2013, "Numerical study of boundary layer flow and heat transfer of Oldroyd -B nanofluid towards a stretching sheet," PLOS ONE, 8.

http://dx.doi.org/10.1371/journal.pone.0069811.

Patil, P., Roy, S., and Chamkha, A., 2009, "Double Diffusive Mixed Convection Flow over a Moving Vertical Plate in The Presence of Internal Heat Generation and a Chemical Reaction," Turkish Journal of Engineering and Environmental Sciences, 33, 193-205. http://dx.doi.org/10.3906/muh-0905-21.

Pop, I., and Tsung, Y., 1998, "A Note on MHD Flow over a Stretching Permeable Surface,” Mechanics Research Communications, 25, 263-269.

Sparrow, E., and Cess, R., 1961, "The Effect of a Magnetic Field on Free Convection Heat Transfer," International Journal of Heat and Mass Transfer, 3, 267-274. 